\title{
Combining sterile and incompatible insect techniques for the population suppression of Drosophila suzukii
}

\author{
K. Nikolouli ${ }^{1,2} \cdot$ F. Sassù ${ }^{1,2} \cdot$ L. Mouton ${ }^{3} \cdot$ C. Stauffer ${ }^{1} \cdot$ K. Bourtzis $^{2}$ (i)
}

Received: 26 August 2019 / Revised: 28 December 2019 / Accepted: 16 January 2020 / Published online: 29 January 2020

(c) The Author(s) 2020

\begin{abstract}
The spotted wing Drosophila, Drosophila suzukii, has recently invaded Europe and the Americas, and it is a major threat for a wide variety of commercial soft fruits both in open field and greenhouse production systems. D. suzukii infests a wide range of ripening fruits, leading to substantial yield and revenue losses. As the application of insecticides close to the harvest period poses great concerns, the development of an efficient environment-friendly control approach to fight $D$. suzukii is necessary. In this study, we exploited the sterile insect technique (SIT) in combination with Wolbachia symbiosis as a population suppression approach that can constitute a potential component of an area-wide integrated pest management program. We aimed to establish a combined SIT/incompatible insect technique (IIT) protocol that would require lower irradiation doses as a complementary tool for D. suzukii management. Two D. suzukii lines trans-infected with the Wolbachia wHa and $w$ Tei strains were irradiated at doses four times less than usual (e.g., $45 \mathrm{~Gy}$ ), and the egg hatching and adult emergence were determined. Our results indicated that $w \mathrm{Ha}$ and $w$ Tei females as well as $w \mathrm{Ha}$ males were sterile at this low dose. The longevity, adult emergence and flight ability of adults were evaluated, and no major effect caused by irradiation was detected. Our data indicate that a SIT/IIT protocol can be a competent approach for D. suzukii management.
\end{abstract}

Keywords Wolbachia $\cdot$ Spotted wing Drosophila $\cdot$ Biological control $\cdot$ Area-wide integrated pest management

\section{Key message}

- Wolbachia-trans-infected D. suzukii lines were irradiated with 45-60-90 Gy irradiation doses, and egg hatch rate and $\mathrm{F} 1$ progeny production were significantly affected.

Communicated by A. Biondi.

Electronic supplementary material The online version of this article (https://doi.org/10.1007/s10340-020-01199-6) contains supplementary material, which is available to authorized users.

K. Bourtzis

K.Bourtzis@iaea.org

1 Department of Forest and Soil Sciences, Boku, University of Natural Resources and Life Sciences, Vienna, Austria

2 Insect Pest Control Section, Joint FAO/IAEA Division of Nuclear Techniques in Food and Agriculture, Wagramerstrasse 5, PO Box 100, 1400 Vienna, Austria

3 Laboratoire de Biométrie et Biologie Evolutive, CNRS, Université de Lyon, Université Claude Bernard Lyon 1, 69100 Villeurbanne, France
- Longevity emergence rate and flight ability were not affected after irradiation at $45 \mathrm{~Gy}$.

- A combined SIT/IIT strategy is proposed for the population suppression of D. suzukii populations as part of an area-wide integrated pest management program.

\section{Introduction}

The spotted wing Drosophila (SWD), Drosophila suzukii (Matsumura) (Diptera: Drosophilidae), is an invasive species originated from Asia that has been recognized as a major concern in agriculture since its first confirmed records in Europe and North America in 2008 (Calabria et al. 2012; Hauser 2011). Predictive models indicate that Australia and Africa offer suitable environmental conditions for potential future invasion of the species (Dos Santos et al. 2017). The constant invasion in new areas has rapidly escalated this pest into a significant global threat for several commercial fruit crops (Asplen et al. 2015; Bolda et al. 2010; Calabria et al. 2012; Cini et al. 2012; Deprá et al. 2014; Walsh et al. 2011). Although different invasion routes have been reported for the 
European and North American populations, the detection of D. suzukii in these two continents was simultaneous, thus supporting the notion that worldwide trade practices are its main dispersal source (Fraimout et al. 2017).

Unique morphological and biological traits of $D$. suzukii, including the female's serrated ovipositor, the short generation time and the ability to adapt in wide temperature and humidity ranges, advanced the dispersal potential and establishment of this pest globally (Hamby et al. 2016; Lee et al. 2011; Sánchez-Ramos et al. 2019a, b; Tochen et al. 2014, 2016; Wong et al. 2018). A series of soft-skinned fruits, both cultivated and wild, serves as trophic niches and oviposition substrates for D. suzukii, and this polyphagy behavior is a key factor for the invasion success of the fly (Lee et al. 2011; Poyet et al. 2015). The high availability of soft fruit crops and their different ripening times throughout the year, combined with the presence of wild hosts, assisted not only in the invasion process, but also in the exceptionally fast adaptation of the fly in the new habitats (Cini et al. 2012; Poyet et al. 2015; Rota-Stabelli et al. 2013; Santoiemma et al. 2019; Tait et al. 2018). As a result the economic impact in the agricultural areas and fruit industry has been enormous (Bolda et al. 2010; De Ros et al. 2015; Mazzi et al. 2017). Extended yield and revenue losses have been reported, while the monitoring and management investments are an additive factor in the economic implications that farmers and companies need to face (DiGiacomo et al. 2019; Goodhue et al. 2011; Mazzi et al. 2017).

Insecticide applications are currently the front-line method used to control D. suzukii in conventional and organic crop areas (Beers et al. 2011; Bruck et al. 2011; Grassi et al. 2011; Rota-Stabelli et al. 2013; Sial et al. 2019; Van Timmeren and Isaacs 2013). Application of insecticides close to the harvest period poses great concerns for the health of both farmers and consumers (Rota-Stabelli et al. 2013), but also to beneficial arthropods (Desneux et al. 2007). The total number of applications for most insecticides is restricted by regulation but, on the other hand, the short generation time of $D$. suzukii requires frequent applications when fruits are at the ripening stage which needs chemicals with a shorter pre-harvest interval (Cini et al. 2012; Goodhue et al. 2011; Sial et al. 2019). This can result in increased insecticide residuals in fruits and unpredictable impacts on pollinators and other non-target species, including D. suzukii's natural enemies (Iglesias and Liburd 2017; Rota-Stabelli et al. 2013; Roubos et al. 2014; Walsh et al. 2011).

The regulatory limitations governing the classical chemical control methods coupled with the concerns over the risks of their use demonstrate the urgent need to develop an alternative environmentally sound and sustainable method to combat D. suzukii (Cuthbertson et al. 2014; Haye et al. 2016; Nikolouli et al. 2018). Insecticide pest resistance, residuals in food, environmental contamination, outbreaks of secondary pests, and reductions in populations of beneficial insects are only some of the major environmental problems that had been caused by the indiscriminate use of insecticides (Bourtzis et al. 2016). The increasing worldwide demand for pest control methods that are both efficient and friendly to the environment has advanced the sterile insect technique (SIT)-based programs. The SIT is a species-specific method of pest population control that relies on mass-produced insects (only males, if feasible) which are sterilized with ionizing radiation, transferred in the target area and bulkreleased until an overflow ratio has been created (Dyck et al. 2005). The success of the SIT relies on the competence of the sterile males to mate with the wild fertile females leading eventually to substantial progeny decline (Dyck et al. 2005; Knipling 1979). The SIT has been for years the workhorse of a plethora of area-wide integrated pest management (AWIPM) programs since its first application against the New World screwworm fly in the 1950's (Dyck et al. 2005; Vreysen et al. 2006). Ever since, the SIT has been successfully launched worldwide to combat various fruit fly species of economic importance and disease vectors of livestock and humans (Cayol et al. 2002; Munhenga et al. 2016; Pereira et al. 2013; Vreysen et al. 2006).

The performance and competitiveness of the released sterile males are important quality parameters and, if not adequate, they might seal the fate of a SIT program (Calkins and Parker 2005). The irradiation dose required for complete male sterility might have profound implications for the biological quality and male mating competitiveness of the insect, as demonstrated for the fruit flies Ceratitis capitata, Anastrepha ludens and Anastrepha obliqua (Guerfali et al. 2012; Rull et al. 2007; Toledo et al. 2004). Significant knowledge has been gained from SIT applications in fruit flies and invested in the development of a biocontrol method against $D$. suzukii with a SIT component. Lanouette et al. (2017), Krüger et al. (2018) and Sassù et al. (2019) (defined the irradiation doses that sterilize males and females without affecting the emergence, longevity and flight ability of the flies.

As shown for the mosquito vector species Aedes albopictus, the combination of the SIT with the IIT could be a well-suited approach for D. suzukii management, as an alternative method to the irradiation-induced sterility (Nikolouli et al. 2018; Zhang et al. 2015b). The IIT employs the mechanism of cytoplasmic incompatibility (CI) in order to produce conditionally sterile males for the control of insect pest populations and disease vectors. CI induced by symbiotic bacteria such as Wolbachia has been studied thoroughly for its potential as a pest population control strategy and has already been exploited against mosquito species in semi-field and field trials (Atyame et al. 2015; Laven 1967; Mains et al. 2019; O'Connor et al. 2012). However, natural 
Wolbachia infections do not occur in all insect species or may not induce CI in their hosts. Trans-infections using embryo microinjections permit host infections with exogenous Wolbachia strains capable of inducing CI (Hughes and Rasgon 2014; Zabalou et al. 2004). Following the proven record of success in tephritid fruit flies (Apostolaki et al. 2011; Zabalou et al. 2004), Cattel and his colleagues (2018) applied this trans-infection approach in D. suzukii. Previous studies reported that the natural Wolbachia infection in $D$. suzukii ( $w$ Suz) shows a variable infection frequency and it is not able to induce strong CI in its host (Cattel et al. 2016; Hamm et al. 2014). Therefore, two Wolbachia strains ( $w \mathrm{Ha}$ and $w$ Tei) acquired by other Drosophila species were microinjected into D. suzukii embryos and stable trans-infected lines were established in the laboratory. In both lines, Wolbachia induced strong CI (95.57\% and 96.46\%, respectively) that was not rescued by the $w$ Suz strain thus giving rise in two promising candidates for a combined SIT/IIT strategy in D. suzukii (Cattel et al. 2018; Nikolouli et al. 2018). The mechanism of this approach has been previously dissected against mosquito vectors (Bourtzis et al. 2016; Lees et al. 2015; Zhang et al. 2015a, b, 2016; Zheng et al. 2019), and it can bear promising results if a low radiation dose to ensure female sterility is applied in flies infected with a Wolbachia strain that induces CI. In such a system, bisexual releases would be feasible since the Wolbachia-infected females would be sterile and the risk of population replacement would be avoided (Lees et al. 2015; Zhang et al. 2015b). In addition, the released males would be able to introduce sterility in the wild population through the combined action of radiation and $\mathrm{CI}$.

In the present study, we aimed to develop a combined SIT/IIT protocol which could potentially be used for the population suppression of D. suzukii. The two Wolbachia candidate strains ( $w \mathrm{Ha}$ and $w$ Tei) suggested by Cattel et al. (2018) were used and three low irradiation doses were evaluated. The effect of the Wolbachia infection and irradiation on the adult emergence, longevity and flight ability were also assessed.

\section{Materials and methods}

\section{D. suzukii lines and rearing conditions}

Four lines harboring the same genetic background from France, but with different infection status were obtained in a previous study by Cattel and colleagues (2018) through microinjections of Wolbachia strains from other Drosophila species into D.suzukii; a Wolbachia-free line (un-Fr), a $w S u z$-infected line ( $w S u z-F r)$ and two trans-infected lines ( $w \mathrm{Ha}-\mathrm{Fr}$ and $w \mathrm{Tei}-\mathrm{Fr}$ ). These two exogenous Wolbachia strains, $w \mathrm{Ha}$ and $w$ Tei, were shown to induce high CI levels in D. suzukii trans-infected lines despite the presence of the natural Wolbachia infection wSuz (Cattel et al. 2018). All these lines were kindly provided by the Laboratory of Biometry and Evolutionary Biology, University Lyon 1, France.

A naturally infected $w$ Suz line (hereinafter mentioned as "wSuz-IPCL") was also obtained from the Agricultural Entomology Unit of the Edmund Mach Foundation in San Michele All'Adige, Trento Province, Italy and maintained in laboratory conditions at the IPCL for 55 generations before its use in this study. A Wolbachia-free line (hereinafter mentioned as "uninfected") was obtained after treatment of the $w$ Suz-IPCL line with $0.25 \mathrm{mg} \mathrm{ml}^{-1}$ tetracycline added in the diet as used in Cattel et al. 2016. The tetracycline treatment lasted for four consecutive generations. In the next two generations, the flies were reared in a diet "inoculated" with $w$ Suz male feces to restore their gut-associated microbial community. After their gut microbiota recovery, the flies were reared in normal standard diet. The absence of Wolbachia was confirmed by PCR targeting the WSpec 16S rDNA region (Werren and Windsor 2000). The WspecF 5'-CAT ACC TAT TCG AAG GGA TAG-3' and WspecR 5'-AGC TTC GAG TGA AAC CAA TTC-3' primers used for the PCR reaction amplify an approximately 440-bp fragment. The amplification reaction mixture contained $1 \mathrm{X}$ Taq PCR Master Mix kit (QIAGEN, Cat No./ID: 201445), which is a premixed solution consisting of Taq DNA Polymerase, PCR Buffer and dNTPs. In addition, $1 \mu \mathrm{M}$ of each primer, $1 \mu \mathrm{L}$ of DNA template and deionized sterile water to a final volume of $25 \mu \mathrm{l}$ were added. The PCR cycle conditions included an initial step at $94{ }^{\circ} \mathrm{C}$ for $3 \mathrm{~min}$, followed by 35 cycles of $94{ }^{\circ} \mathrm{C}$ for $45 \mathrm{~s}, 55^{\circ} \mathrm{C}$ for $45 \mathrm{~s}, 72{ }^{\circ} \mathrm{C}$ for $1 \mathrm{~min}$, and a final elongation step at $72^{\circ} \mathrm{C}$ for $10 \mathrm{~min}$.

All D. suzukii lines were reared in a carrot-based diet containing: $1 \%$ agar, $3.75 \%$ sugar, $3.75 \%$ carrot powder (Kanegrade Ltd), $1.5 \%$ yellow corn meal, $2.25 \%$ inactive dry yeast and $0.5 \%$ propionic acid, and maintained at a room with $23-24{ }^{\circ} \mathrm{C}$ temperature, $45-50 \%$ humidity and natural light conditions.

\section{Cytoplasm introgression}

Introgression experiments were performed to align all Wolbachia strains under a common genetic background. Females from $w \mathrm{Ha}-\mathrm{Fr}, w$ Tei-Fr and $w$ Suz-Fr lines were crossed with males from the $w$ Suz-IPCL line. The female offspring of these crosses were then backcrossed to $w$ Suz-IPCL males for a total of eight generations. After the final backcrossing, the three lines, hereinafter mentioned as " $w \mathrm{Ha}$ " $w$ Tei" and "wSuz", were checked with PCR to confirm Wolbachia presence, as described above. Multi-Locus Sequence Typing (MLST) analysis of Wolbachia was performed by PCRs targeting six genes (wsp, gatB, $\operatorname{cox} A, h c p A, f t s Z, f b p A)$ as described in Baldo et al. (2006) in order to verify that the 
Wolbachia strain was the one expected in the lines. PCR products were sequenced, and sequences were aligned using the ClustalW algorithm in the BioEdit v.7.0.5 software (Hall 1999).

\section{Life-history traits of $D$. suzukii lines}

Unless otherwise stated, in all experiments freshly emerged adults were sexed and placed separately in vials with standard diet until aged 5-6 days old. At that time point, the adults were used for the assays described below. All experiments were performed at constant laboratory conditions as described above.

To estimate the effect of Wolbachia on the fecundity and hatch rate of the lines, we placed 10 virgin males and 10 virgin females in a standard diet vial and allowed them to mate for $48 \mathrm{~h}$. Males were then removed, and females laid eggs individually in Petri dishes containing a substrate of raspberry-juice agar. The females were transferred daily to a fresh substrate until three changes had been completed $(72 \mathrm{~h}$ of egg laying in total). The number of eggs was counted daily, and the hatching was recorded $48 \mathrm{~h}$ after egg laying to ensure that all eggs were given enough time to hatch. Three replicates were performed for each of the four lines. Fecundity was calculated as the average number of eggs laid per female, and hatch rate was determined as the number of hatched eggs per the total number of eggs laid.

Subsequently, the larvae were placed in a Petri dish with standard diet and allowed to pupate. The pupal weight was measured 1 day before adult emergence. Pupae from each line were sorted into 10 replicate groups with 5 pupae in each group and they were weighted.

To check whether the progeny balance is affected by Wolbachia, we placed 10 virgin males and 10 virgin females in a standard diet vial and allowed them to mate for $48 \mathrm{~h}$. Males were removed, and females laid eggs individually in vials with standard diet for $48 \mathrm{~h}$. Females were then transferred to fresh vials and allowed again to lay eggs for another $48 \mathrm{~h}$. Adult emergence was recorded daily, and sex ratio was determined as proportion of males per total number of adults. Three replicates were performed per line.

Adult longevity was assessed by placing 15 newly emerged males and females separately in vials with standard diet. Mortality was monitored daily and until all adults were dead. The experiment was performed in triplicates per line per sex.

\section{Cytoplasmic incompatibility $(\mathrm{Cl})$ assays}

The CI expression levels of $w \mathrm{Ha}$ and $w$ Tei males were checked in crosses with either wSuz or uninfected females. All CI crosses were single, and we used 2-to-3-day-old virgin males and 5-to-6-day-old virgin females. The couple was placed in a vial with a fresh raspberry and allowed to mate for $24 \mathrm{~h}$. The vials were inspected multiple times during the day, and the couples that mated for at least $15 \mathrm{~min}$ were recorded and used downstream. The couples for which no mating was observed were discarded. Males were then removed, and females were allowed to lay eggs individually in a raspberry-juice agar substrate for $48 \mathrm{~h}$. After that, females were transferred again individually in a new Petri dish for another $48 \mathrm{~h}$. Only females that laid at least 20 eggs in total were included in the analysis. At least 19 repetitions were performed for each cross type (compatible and incompatible).

The corrected index of $\mathrm{CI}\left(\mathrm{CI}_{\text {corr }}\right)$ (Poinsot et al. 1998) was used to minimize the variation effect that the natural embryonic mortality could have on the CI level estimation. This mortality is not related to CI, and it is defined by the compatible cross scheme. $\mathrm{CI}_{\text {corr }}$ is calculated as: $\mathrm{CI}_{\text {corr }}=\left[\left(\mathrm{CI}_{\text {obs }}-\mathrm{CCM}\right) /(100-\mathrm{CCM})\right] \times 100$, where $\mathrm{CI}_{\text {obs }}$ is the percentage of unhatched eggs in the incompatible cross, and CCM is the mean mortality observed in the control crosses.

\section{Effect of male age on $\mathrm{Cl}$ levels}

Male age is a factor known to potentially reduce the CI levels. We investigated the impact of male age on the CI intensity by crossing $w \mathrm{Ha}$ and $w$ Tei males with $w$ Suz or uninfected females. Males and females were sexed and placed separately in vials with standard diet. The males that were used in the crosses were $2-3,5-6$ or 9-10 days old, while in all cases the females were 5-6 days old. Mass crosses of ten males and ten females were performed and allowed to mate for $48 \mathrm{~h}$. Males were removed, and females were placed in a common cage to lay eggs in raspberry-juice agar for $48 \mathrm{~h}$. Control crosses of the uninfected and $w$ Suz lines were used to quantify the effect of male age in the absence of CIinducing Wolbachia strains. The $\mathrm{CI}$ corrected index $\left(\mathrm{CI}_{\text {corr }}\right)$ was used again for the assessment of the male age effect. Six replicates were performed for each cross type.

\section{Effect of irradiation dose on egg hatch rate}

A ${ }^{60}$ Co irradiator (Gamma Cell-220, Nordion, Canada) was used for the irradiation of $w \mathrm{Ha}$ and $w$ Tei pupae. The pupae of each strain were collected one day before emergence, placed in a $60 \times 15 \mathrm{~mm}$ Petri dish and irradiated. Based on previous knowledge about the irradiation dose required for sterilizing completely $D$. suzukii females (Krüger et al. 2018; Lanouette et al. 2017; Sassù et al. 2019), the doses tested here were 45, 60 and $90 \mathrm{~Gy}$. After irradiation, the pupae were placed in a cage and the emerged adults were collected next day. The collection of irradiated adults lasted for $24 \mathrm{~h}$ after irradiation and all adults that emerged later 
were discarded, as they were considered young at the time of irradiation. Irradiated males and females were sexed and placed separately in vials with standard diet for 5-6 days. Virgin fertile $w$ Suz males and females were also collected simultaneously. To assess the egg hatch rate of the $w \mathrm{Ha}$ and $w$ Tei adults, fifteen irradiated males and females from each strain were crossed with fifteen fertile $w$ Suz females and males, respectively, and allowed to mate and oviposit in raspberry-juice agar substrate for $24 \mathrm{~h}$. The oviposition substrate was replaced daily for three consecutive days. All control crosses of the fertile $w$ Suz, $w$ Ha and $w$ Tei adults were also performed. The eggs were counted and transferred in Petri dishes with standard diet to ensure that all nutrients required for the larval development were supplied. Egg hatching was recorded $48 \mathrm{~h}$ after oviposition. The experiment was performed in two different time points. At first the doses 60 and 90 Gy were applied, and three replicates per cross type, per dose were performed. At the next generation, the doses 45 and $60 \mathrm{~Gy}$ were applied and three replicates per cross type, per dose were performed. The $60 \mathrm{~Gy}$ irradiation dose was performed twice as a reference to normalize any effect of time in our data.

Three pieces of Gafchromic ${ }^{\circledR} \mathrm{HD}-\mathrm{V} 2$ dosimetry films (International Specialty Products, NJ, USA) $(10 \times 10 \mathrm{~mm})$ were centered on top of the Petri dish before the irradiation. Twenty-four hours after irradiation, the films were read by a Radiochromic reader (FWT-92D, Far West Technology, Inc., Goleta, CA, USA) to confirm the irradiation dose that the pupae had received. Dosimetry was performed according to the manual and all readings were within the $95 \%$ confidence intervals (Gafchromic ${ }^{\circledR}$ Dosimetry System for SIT. Standard Operating Procedure 2004).

\section{Effect of irradiation on adult emergence rate}

$w \mathrm{Ha}$ and $w$ Tei pupae were irradiated at 0 and $45 \mathrm{~Gy}$ as described above. The pupae were clustered in groups of fifteen, placed in vials and left to emerge. Non-irradiated $w \mathrm{Suz}$ pupae were also included as control. The number of emerged adults and their sex were recorded in all cases. Three replicates were performed for each line.

\section{Effect of irradiation on adult longevity}

The longevity of irradiated and non-irradiated adults was assessed both for males and females. $w \mathrm{Ha}$ and $w$ Tei pupae were irradiated at 0 and $45 \mathrm{~Gy}$, and freshly emerged adults were sexed and placed in vials containing an agar-sugar substrate (1\% agar, $10 \%$ sugar, $1 \%$ yeast dissolved in water). Fifteen adults were included per vial, and three replicates per line, treatment, and sex were performed.

\section{Effect of irradiation on flight ability}

Irradiated at $45 \mathrm{~Gy}$ and non-irradiated $w \mathrm{Ha}$ and $w$ Tei pupae were used to assess the effect of irradiation on the adult flight ability. $w$ Suz non-irradiated pupae were also included as control. After irradiation, pupae were placed at the bottom of an open Petri dish. A black plexiglass tube was adjusted over the Petri dish and the tube's internal site was coated with unscented talcum powder to prevent flies from crawling out of the tube (FAO/IAEA/USDA 2014). Flies were periodically aspirated from the vicinity of the tubes to avoid falling-back into the tubes. Five replicates with 15 pupae each were set up per treatment.

\section{Statistical analysis}

All data were examined for normality using the Shapiro-Wilk normality test. In data sets where the normality assumption was violated, nonparametric tests were applied. Analysis of variance (ANOVA) was used to examine the significance of interactions between factors. Interactions that were not significant were excluded and models were simplified. Lsmeans (Lenth 2016) and multcomp (Hothorn et al. 2008) packages were used for the pairwise comparisons of the fitted model estimates. In all datasets, the Wolbachia strain was included as a fixed factor and replicates as a random factor. Pupal weight data were analyzed using a linear mixed-effect model and they were square transformed to improve normality of the residual errors. A GLMM (binomial family) was used for the analysis of the CI, age of males' effect, emergence rate, flight ability, and hatching rates. Fecundity and sex ratio data were analyzed with a GLMM (Poisson family). The survivorship curves were calculated using a Kaplan-Meier approach (survfit package) (Kaplan and Meier 1958). All statistical analyses were performed using R version 3.5.2 (R Core Team 2018). The package lme 4 was used for all mixed models (Bates et al. 2015). The package survival was used for modeling the longevity data (Therneau 2015). In all cases the mean \pm standard error is reported. The statistical results for all datasets are available in Online Resource 4.

\section{Results}

\section{Life-history traits of the introgressed D. suzukii lines}

We did not detect any effect of the infection status on the fecundity of the lines after $72 \mathrm{~h}$ of egg laying. Despite the fact that the mean egg production of the uninfected line was considerably higher $(42.7 \pm 4.58)$ compared to the $w \mathrm{Ha}(22.7 \pm 2.86), w \mathrm{Tei}(22 \pm 2.9)$ and $w \operatorname{Suz}(26 \pm 3.32)$ lines (Online Resource 1a), the differences among the lines 
were not significant (uninfected- $w$ Ha GLMM: $z=-1.608$, $p=0.374$; uninfected- $w$ Tei GLMM: $z=-1.833, p=0.258$; uninfected- $w$ Suz GLMM: $z=-1.093, p=0.694)$. Absence of any effect was also observed on the hatch rates of the lines (Online Resource 1b), although the uninfected line showed marginally higher hatch rate $(91.5 \% \pm 1.67)$, compared to the $w \mathrm{Ha}, w \mathrm{Suz}$ and $w$ Tei lines $(86 \% \pm 5.91$, $84.5 \% \pm 6.21$ and $77.9 \% \pm 7.48$, respectively).

The pupal weight data showed that the uninfected and the $w$ Suz lines had higher average weight $(17.2 \pm 0.24 \mathrm{mg}$ and $16.7 \pm 0.43 \mathrm{mg}$, respectively) compared to the $w \mathrm{Ha}$ and $w$ Tei lines $(14.7 \pm 0.52 \mathrm{mg}$ and $15.5 \pm 0.39 \mathrm{mg}$, respectively) (Online Resource 2). The data showed that the infection status was related to the pupal weight $\left(F_{3,36}=7.509, p=0.0005\right)$.

The infection status did not result in any sex ratio imbalances in the four $D$. suzukii lines. No significant differences were observed among the lines (Kruskal-Wallis; $\left.\chi^{2}=6.9361, d f=3, p=0.073\right)$. The sex ratio in the uninfected line was $0.487 \pm 0.03$, in the $w \mathrm{Ha}$ line $0.506 \pm 0.03$, in the $w$ Suz line $0.557 \pm 0.02$ and in the $w$ Tei line $0.498 \pm 0.03$.

The presence of Wolbachia did not appear to impact the adult longevity. Due to the continuous supply of an energy source and water, the mortality rates were extremely low during the first 40 days of the experiment. Therefore, the mortality was recorded daily up to 58 days and after that, the experiment was discontinued, since the evaluation of such long longevity times is untenable from an application point of view. Both females (log-rank test; $\chi^{2}=3.1, d f=3$, $p=0.4$ ) and males (log-rank test; $\chi^{2}=5.2, d f=3, p=0.2$ ) presented the same survival probability, regardless of the infection status (Online Resource 3a, b).

\section{$\mathrm{Cl}$ expression levels}

We determined the CI levels in single-pair crosses that included males infected either with $w \mathrm{Ha}$ or $w$ Tei and females uninfected or infected with $w$ Suz (Fig. 1). Our results showed that $w \mathrm{Ha}$ induces strong $\mathrm{CI}$ when crossed with uninfected or $w$ Suz-infected females $(98.9 \% \pm 0.68$ and $98.5 \% \pm 0.74, \mathrm{CI}_{\text {corr }}$, respectively). The difference between uninfected and $w$ Suz-infected females was not statistically significant (GLMM: $z=-0.305, p=1$ ) indicating that CI induced by $w \mathrm{Ha}$ is not rescued by the natural $w$ Suz infection. On the other hand, crosses with $w$ Tei males presented significantly lower $\mathrm{CI}$ levels compared to the respective ones with $w \mathrm{Ha}$ (un $\varnothing \times w$ Tei $\hat{\sigma}-$ un $q \times w \mathrm{Ha} \hat{\circ}$ GLMM: $z=-6.014$, $p=<1 \mathrm{e}-05 ; w \mathrm{Suz} q \times w$ Tei $\hat{\sigma}-w \mathrm{Suz} q \times w \mathrm{Ha} \hat{\delta}$ GLMM: $z=6.829, p=<1 \mathrm{e}-05)$. The $\mathrm{CI}_{\text {corr }}$ values were $64.8 \% \pm 8.37$ for crosses with uninfected females and $67.4 \% \pm 5.6$ for crosses with $w$ Suz-infected females.

\section{Effect of male age on $\mathrm{Cl}$ levels}

In our control crosses, there was no significant difference among the different age groups of the males. We observed significant differences in the $\mathrm{CI}_{\text {corr }}$ levels in crosses with $w \mathrm{Ha}$ males that were dropping as the age of the males was increased (Fig. 2a). In crosses between uninfected females and $w \mathrm{Ha}$ infected males, the $\mathrm{CI}_{\text {corr }}$ was $97.7 \% \pm 1.25$ in males aged $2-3$ days old and dropped to $65.3 \% \pm 11.26$ in
Fig. 1 Cytoplasmic incompatibility levels estimated in individual crosses in D. suzukii lines. In all crosses the males were $2-3$ days old and the females were 5-6 days old. The corrected index of $\mathrm{CI}\left(\mathrm{CI}_{\text {corr }}\right)$ was used to account for the basal embryonic mortality and estimate only the CI-related mortality. A GLMM (binomial family) analysis was performed to determine the differences between the crosses. (un $9 \times$ un ${ }^{\lambda}: \mathrm{N}=24 ; w \operatorname{Suz} q \times w \operatorname{Suz}_{\bigcirc}{ }^{\wedge}:$ $\mathrm{N}=20 ; w \operatorname{Suz}+\times w \mathrm{Ha}^{\top}:$ $N=22$; un우 $\times w \mathrm{Ha}^{\top}: \mathrm{N}=20$; $w$ Suz 우 $\times w$ Tei ${ }^{\wedge}: \mathrm{N}=26$; un우 $\times w \operatorname{Tei}^{\lambda}: N=19$; Confidence level used: 0.95 , alpha $=0.05$ )

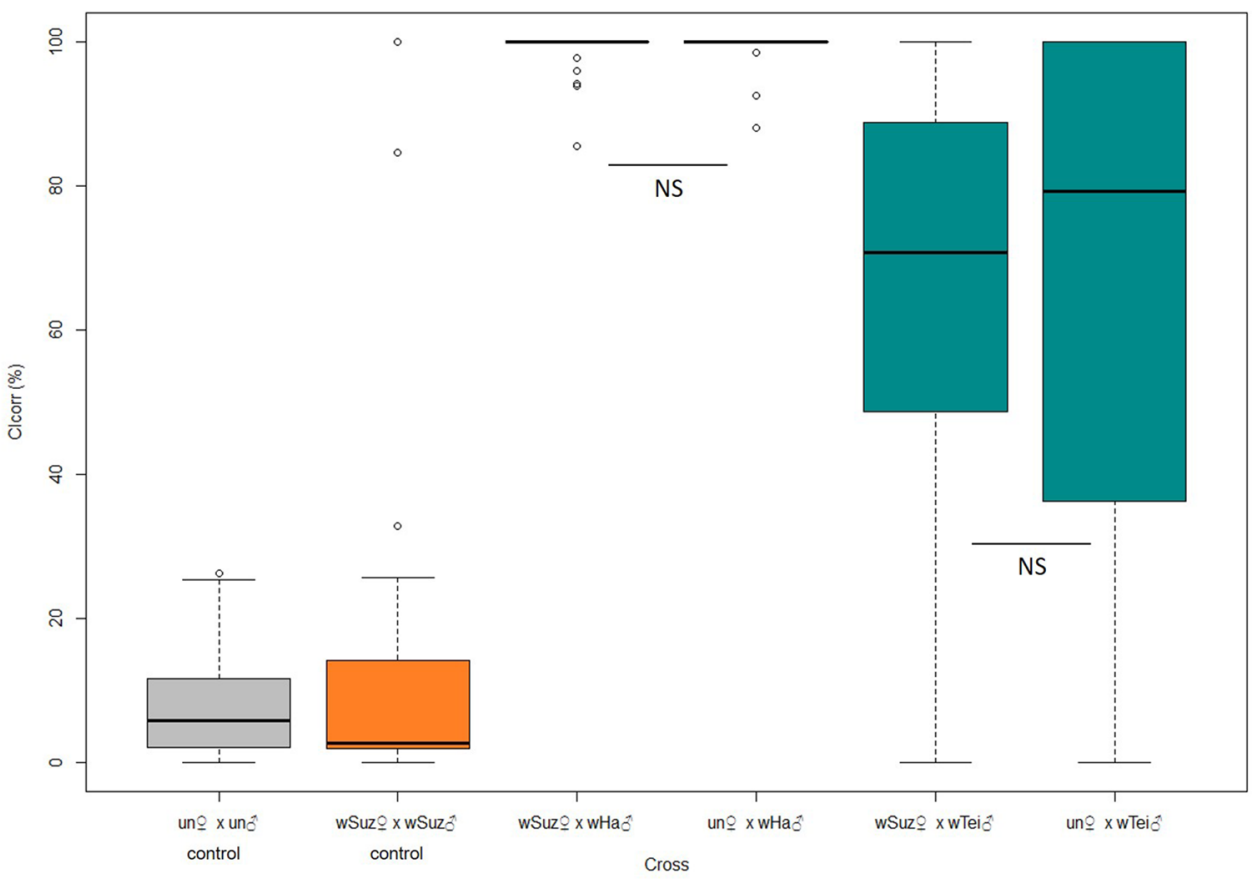


Fig. 2 Effect of male age on CI levels in D. suzukii crosses. In all crosses the females were 5-6 days old. a Crosses with $w$ Ha infected males; $\mathbf{b}$ crosses with $w$ Tei infected males. The corrected index of $\mathrm{CI}\left(\mathrm{CI}_{\text {corr }}\right)$ was used to account for the basal embryonic mortality and estimate only the CI-related mortality. A GLMM (binomial family) analysis was performed to determine the differences between the crosses. $* * * p<.001, * * p<.01, * p<.05$ (Confidence level used: 0.95, alpha $=0.05 ; N=60$ )
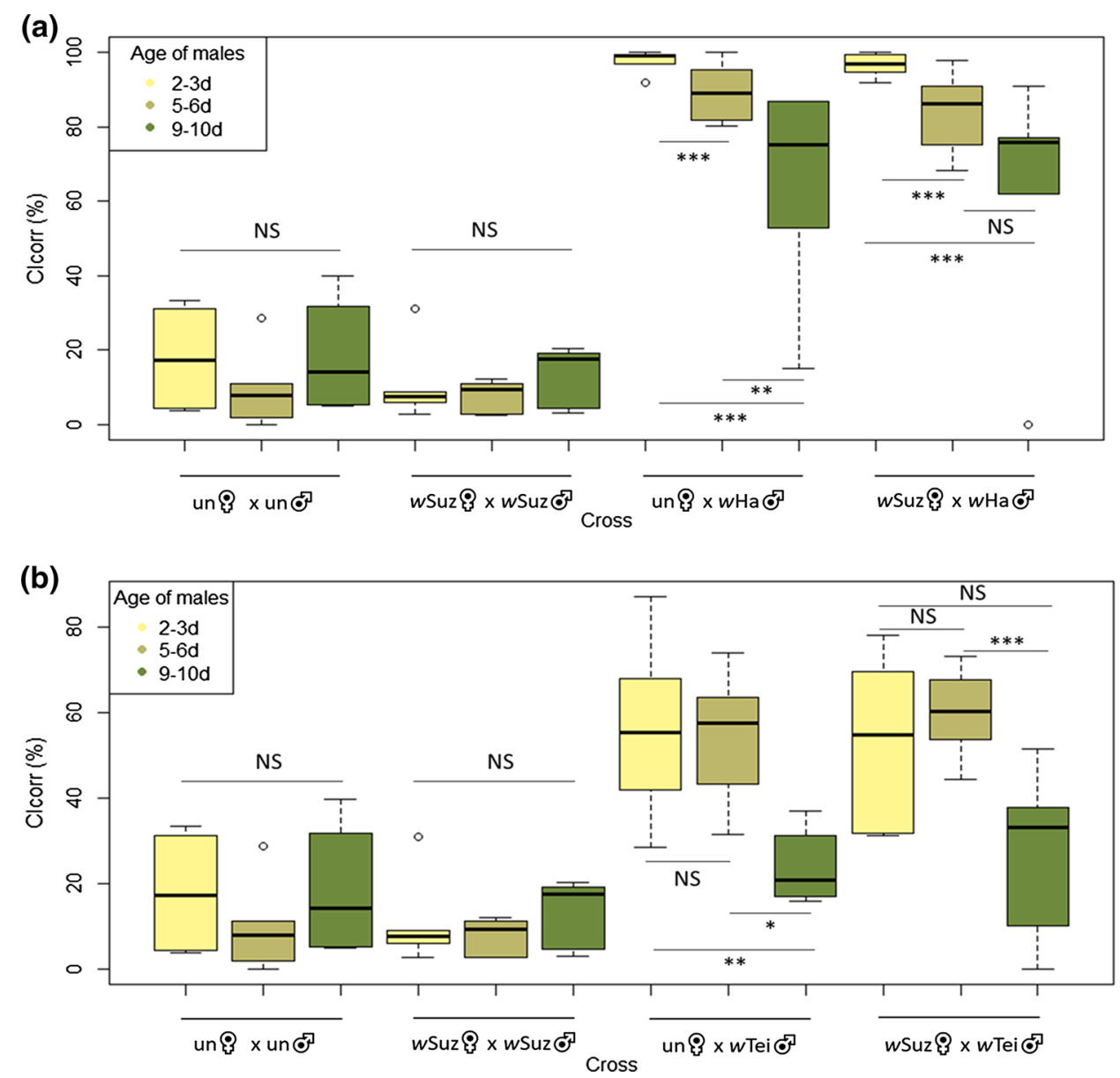

males aged 9-10 days old (Fig. 2a). A notable decrease was also observed in crosses with $w$ Suz-infected females $\left(96.6 \% \pm 1.31 \mathrm{CI}_{\text {corr }}\right.$ in 2-to-3-day-old $w \mathrm{Ha}$ males and $63.5 \% \pm 13.2 \mathrm{CI}_{\text {corr }}$ in 9-to-10-day-old $w$ Ha males) thus indicating an effect of the male age on the CI intensity of the $w \mathrm{Ha}$ line. The age of males affected the CI intensity also in crosses with $w$ Tei males. $\mathrm{CI}_{\text {corr }}$ dropped from $56 \% \pm 8.28$ (2 to 3 days old) to $23.8 \% \pm 3.42$ (9 to 10 days old) and from $53.3 \% \pm 7.87$ ( 2 to 3 days old) to $27.6 \% \pm 7.71$ ( 9 to 10 days old) in crosses with uninfected and $w$ Suz-infected females, respectively (Fig. 2b).

\section{Effect of irradiation dose on egg hatch rate}

Egg hatch rate data revealed an effect of radiation on the progeny production in the Wolbachia-infected lines (Table 1). In the control crosses of the $w \mathrm{Ha}, w$ Tei and $w$ Suz lines (which consisted of non-irradiated, fertile females and males of each line), the hatching rate was $93.8 \% \pm 2.85,92.3 \% \pm 2.97$, and $88.4 \% \pm 3.98$, respectively. When irradiated $w \mathrm{Ha}$ females were crossed with fertile $w$ Suz males, no eggs were laid at 60 and $90 \mathrm{~Gy}$, while we collected only 6 eggs at $45 \mathrm{~Gy}$ which did not hatch (Fig. 3a). The irradiated $w$ Tei females laid 1 egg at 45 Gy that did not hatch, 6 eggs at 60 Gy out of which only one hatched, but the larva died before pupation and 1 nonhatched egg at 90 Gy (Fig. 3b). Significant decrease in egg hatch rate was also observed in the crosses between irradiated males and fertile $w$ Suz females. When $w$ Ha males were irradiated at $45 \mathrm{~Gy}$ and crossed with fertile $w \mathrm{Suz}$ females, only $1 \mathrm{egg}$ hatched out of the 782 collected eggs $(0.13 \%)$. At $60 \mathrm{~Gy}$ we collected 926 eggs and only 2 of them hatched $(0.23 \%)$ and at $90 \mathrm{~Gy}$ the egg hatching was $0.33 \%$ ( 1 hatched out of the 300 collected eggs) (Fig. 4a). In all the above cases involving irradiated $w$ Ha males, the larvae died before the pupation stage (Table 1 ). In the case of $w$ Tei irradiated males, at 45 Gy the egg hatching was $1.1 \%$ (17 out of 1537 eggs hatched), at 60 Gy it was $2.4 \%$ (25 out of 1048 eggs hatched) and at $90 \mathrm{~Gy}$ it was $1 \%$ (3 out of 300 eggs hatched) (Fig. 4b). The number of F1 progeny (pupae and emerged adults) coming from crosses with irradiated $w$ Tei males is described in Table 1. 
Table 1 Effect of irradiation on the egg hatching and adult emergence of the $w \mathrm{Ha}$ and $w$ Tei D. suzukii lines, when females or males of these lines are irradiated

\begin{tabular}{|c|c|c|c|c|c|c|}
\hline Cross & $\begin{array}{l}\text { Irradiation } \\
\text { dose (Gy) }\end{array}$ & Number of eggs & $\begin{array}{l}\text { Number of } \\
\text { hatched eggs }\end{array}$ & $\begin{array}{l}\text { Number } \\
\text { of pupae }\end{array}$ & $\begin{array}{l}\text { Emerged } \\
\text { females }\end{array}$ & Emerged males \\
\hline$w \mathrm{Ha}+\times w \mathrm{Ha} \hat{\sigma}$ & 0 & 600 & 563 & 453 & 198 & 190 \\
\hline$w \operatorname{Tei} \phi \times w \operatorname{Tei} \hat{\sigma}$ & 0 & 600 & 554 & 418 & 200 & 192 \\
\hline$w \operatorname{Suz}+\times w \operatorname{Suz} \delta^{\lambda}$ & 0 & 593 & 524 & 435 & 187 & 209 \\
\hline$w \mathrm{Ha} \propto+\times w \mathrm{Suz}_{\hat{O}} \widehat{ }$ & 45 & 6 & 0 & 0 & 0 & 0 \\
\hline$w \mathrm{Ha} q \times w \operatorname{Suz}_{\hat{O}} \hat{}$ & 60 & 0 & 0 & 0 & 0 & 0 \\
\hline$w \mathrm{Ha} q \times w \operatorname{Suz}_{\bigcirc}^{\lambda}$ & 90 & 0 & 0 & 0 & 0 & 0 \\
\hline$w \operatorname{Tei} \phi \times w \operatorname{Suz}_{\bigcirc} \hat{}$ & 45 & 1 & 0 & 0 & 0 & 0 \\
\hline$w \operatorname{Te} i \phi \times w \operatorname{Suz}_{\varnothing} \hat{}$ & 60 & 6 & 1 & 0 & 0 & 0 \\
\hline$w \operatorname{Tei} \phi+\times w \operatorname{Suz}^{\lambda}$ & 90 & 1 & 0 & 0 & 0 & 0 \\
\hline$w \mathrm{Suz}+{ }^{\circ} \times w \mathrm{Ha}^{\wedge}$ & 45 & 782 & 1 & 0 & 0 & 0 \\
\hline$w \mathrm{Suz}+{ }^{\circ} \times w \mathrm{Ha}^{\wedge}$ & 60 & 926 & 2 & 0 & 0 & 0 \\
\hline$w \mathrm{Suz}+\times w \mathrm{Ha}^{\lambda}$ & 90 & 300 & 1 & 0 & 0 & 0 \\
\hline 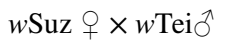 & 45 & 1537 & 17 & 16 & 9 & 5 \\
\hline 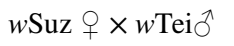 & 60 & 1048 & 25 & 19 & 7 & 11 \\
\hline$w \operatorname{Suz} q \times w \operatorname{Tei}^{\gamma} \hat{\jmath}$ & 90 & 300 & 3 & 1 & 0 & 1 \\
\hline
\end{tabular}

fliers were $82.1 \% \pm 1.83$ (GLMM: $z=0.517, p=0.9844$ ) (Fig. 7).

\section{longevity and flight ability}

The effect of irradiation at $45 \mathrm{~Gy}$ on adult emergence was assessed both for $w \mathrm{Ha}$ and $w$ Tei adults. The emergence rate was significantly influenced by the irradiation dose (Kruskal-Wallis; $\chi^{2}=11.21, d f=4, p=0.0243$ ). We observed significant differences between $w \mathrm{Ha}$ adults irradiated at $45 \mathrm{~Gy}$ and $w \mathrm{Ha}$ non-irradiated adults (GLMM: $z=-3.256, p=0.0099$ ), while the differences between irradiated and non-irradiated $w$ Tei adults were not significant (GLMM: $z=1.989, p=0.2709$ ) (Fig. 5).

Adult longevity data showed a significant effect of irradiation on the survival days of both females and males ( (log-rank test: $\chi^{2}=19.2, d f=3, p=2 \mathrm{e}-04$; $\widehat{o} \log$-rank test: $\chi^{2}=27.1, d f=3, p=5 \mathrm{e}-06$ ) (Fig. 6a). Irradiated $w \mathrm{Ha}$ females had significantly shorter longevity times compared to the non-irradiated ones ( $q w \mathrm{Ha}$ irradiated vs. $w$ Ha non-irradiated: $\chi^{2}=9.3, d f=1, p=0.002$ ), while the same was not true for $w$ Tei females ( $q w$ Tei irradiated vs. $w$ Tei non-irradiated: $\chi^{2}=0.3, d f=1, p=0.6$ ). The opposite pattern was observed for the males; the difference was non-significant for the $w$ Ha males ( $\hat{w} w \mathrm{Ha}$ irradiated vs. $w$ Ha non-irradiated: $\chi^{2}=2.2, d f=1, p=0.1$ ), but significant difference was observed for the $w$ Tei males ( $\partial^{\top} w$ Tei irradiated vs. $w$ Tei non-irradiated: $\chi^{2}=18.8, d f=1$, $p=1 \mathrm{e}-05)$ (Fig. 6b).

The flight ability data showed that there was no significant difference between the irradiated and non-irradiated flies. In the $w \mathrm{Ha}$ line, the fliers coming from the irradiated pupae were $98.6 \% \pm 1.42$ and $90.7 \% \pm 3.07$ from the untreated pupae (GLMM: $z=-1.750, p=0.3825$ ). The same result was observed for the $w$ Tei individuals, where the irradiated fliers were $78.5 \% \pm 4.6$ and the control $w$ Tei

\section{Discussion}

Our main goal in this study was to develop a combined SIT and IIT approach which could potentially be used for the control of D. suzukii populations. A previous study determined the optimum irradiation dose for an adequate $D$. suzukii male sterility level (99.67\%) at $200 \mathrm{~Gy}$ (Krüger et al. 2018). In addition, Lanouette and her colleagues (2017) showed that $96 \%$ of sterility can be achieved if males are irradiated at $120 \mathrm{~Gy}$, while full female sterility was achieved at $75 \mathrm{~Gy}$ in both studies. Assessing the effect of high irradiation doses on several quality parameters (emergence rate, flight ability, longevity and sex ratio) did not indicate any alterations or decay in performance in both studies (Krüger et al. 2018; Lanouette et al. 2017).

Given the above promising results and considering the urge to develop a sustainable and environmentally sound approach, we aimed to establish a combined SIT/IIT protocol that would require lower irradiation doses that may not affect the quality of sterile males, as shown for the mosquito vector species Ae. albopictus (Zhang et al. 2015b; Zheng et al. 2019) and would act as a complementary tool for $D$. suzukii management. Based on the female sterility dose suggested by Krüger et al. (2018) and Lanouette et al. (2017), we tested the irradiation doses at 45,60 and $90 \mathrm{~Gy}$ on $w H a$ and $w T e i$ trans-infected individuals and observed complete sterility both for $w \mathrm{Ha}$ and $w$ Tei females at all three doses. Similar encouraging results were also obtained for males $(99.887 \%$ sterility for $w \mathrm{Ha}$ males and $98.9 \%$ sterility for $w$ Tei males at $45 \mathrm{~Gy}$ ). None of the quality control parameters tested at 

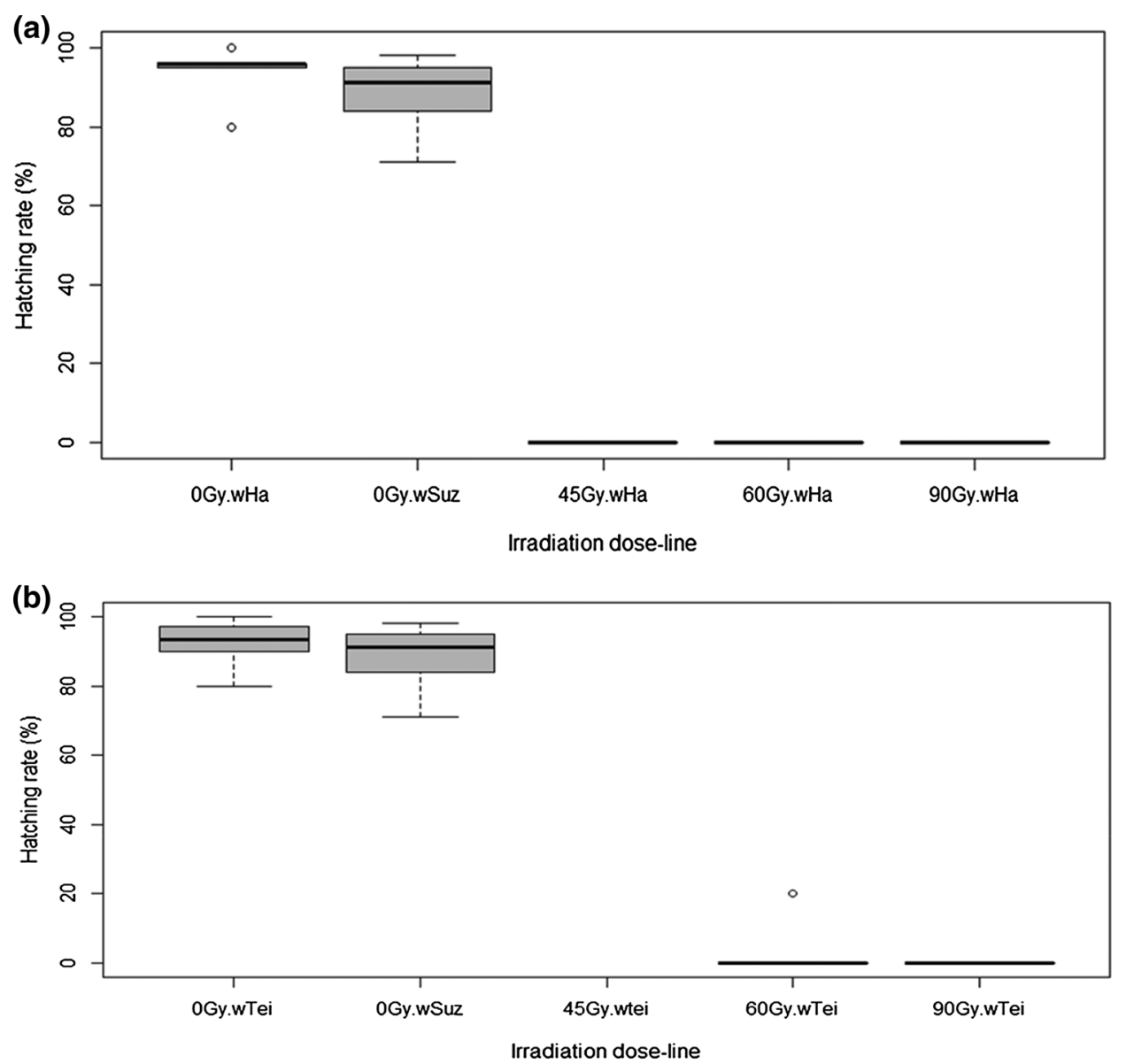

Fig. 3 Hatching rates of crosses between irradiated, Wolbachiainfected females and fertile, wSuz males. a Crosses with $w \mathrm{Ha}$ infected females; (Kruskal-Wallis Chi-squared $=20.334, d f=4$, $p$ value $=0.0004289) ;\left[0\right.$ Gy $w \mathrm{Ha}: w \mathrm{Ha}+{ }^{\circ} \times w \mathrm{Ha} \delta$ (fertile, nonirradiated females and males), 0 Gy.wSuz: $w$ Suz $\circ \times w$ Suz $\delta$ (fertile, non-irradiated females and males), xxGy.wHa: $w \mathrm{Ha} q \times$ $w$ Suz $\hat{\sigma}$ (irradiated $w$ Ha females crossed with fertile $w$ Suz males)], b Crosses with $w$ Tei infected females; (Kruskal-Wallis Chi-

45 Gy was negatively affected, excluding the irradiated wHa adults that were positively affected showing elevated emergence rate compared to the non-irradiated ones.

The noteworthy difference in the irradiation dose between $w$ Suz-infected males, as defined by previous studies (Krüger et al. 2018; Lanouette et al. 2017), and $w \mathrm{Ha}$ and $w$ Tei infected males, as defined by the present study, demonstrates an engagement of the Wolbachia infection in the effect prompted by irradiation on the egg hatching rate. Irradiation is known to induce the formation of free radicals that create dominant lethal mutations in the germ cells (Bakri et al. 2005). The low irradiation dose we determined for the complete sterility of the $w \mathrm{Ha}$ and $w$ Tei lines might suggest a higher susceptibility of the transinfected lines to the oxidative stress caused by irradiation (Monnin et al. 2016). Future research is required to dissect the mechanism underlying the antioxidant capacity of squared $=19.338, d f=4, p$ value $=0.0006743) ;[0$ Gy. $w$ Tei: $w$ Tei + $\times w$ Tei $\delta$ (fertile, non-irradiated females and males), 0 Gy.wSuz: $w$ Suz $q \times w$ Suz $\hat{o}$ (fertile, non-irradiated females and males), xxGy. $w$ Tei: $w$ Tei $q \times w$ Suz $\delta$ (irradiated $w$ Tei females crossed with fertile $w$ Suz males)]. Pupae were irradiated at 45, 60 and $90 \mathrm{~Gy}$. All adults used for the crosses were 5-6 days old. A GLMM (binomial family) analysis was performed to determine the differences between the crosses. alpha $=0.05$

the two trans-infected lines. The increased emergence rate observed for the irradiated $w \mathrm{Ha}$ adults could be attributed to the hormesis hypothesis. Hormesis has been described as the stimulatory outcome observed after mild or sublethal stress levels and it has long been realized in insects (Le Bourg 2010; Cutler 2013). Low stress levels can have beneficial effects on several biological traits of insects, and this could explain why we noticed a performance gain in terms of adult emergence in the 45 Gy-irradiated $w \mathrm{Ha}$ line and not in the control one. The increased adult emergence after a low irradiation dose for the $w \mathrm{Ha}$ line is an add-in value for the combined SIT/IIT approach for D. suzukii.

The presence or absence of Wolbachia, as well as the different Wolbachia strains and the host genome can lead in phenotypic variations across host species. Martinez and colleagues (2017) showed that the antiviral protection phenotype exerted by the same symbiont was mostly dependent 
Fig. 4 Hatching rates of crosses between irradiated, Wolbachiainfected males and fertile, $w$ Suz females. a Crosses with $\mathrm{wHa}$ infected males; (Kruskal-Wallis Chi-squared $=18.814, d f=4, p$ value $=0.0008548)$; [0 Gy. $w$ Ha: $w \mathrm{Ha}+\times \times w \mathrm{Ha}$ ○ึ (fertile, nonirradiated females and males), 0 Gy. $w$ Suz: $w \operatorname{Suz} q \times w$ Suz $\delta$ (fertile, non-irradiated females and males), xxGy.wHa: $w$ Suz $q$ $\times w \mathrm{Ha}$ ○े (fertile $w$ Suz females crossed with irradiated $w \mathrm{Ha}$ males)], b Crosses with $w$ Tei infected males; (Kruskal-Wallis Chi-squared $=17.995, d f=4, p$ value $=0.001237) ;[0$ Gy. $w$ Tei: $w$ Tei $q \times w$ Tei $\widehat{\partial}$ (fertile, nonirradiated females and males), 0 Gy. $w$ Suz: $w$ Suz $q \times w$ Suz 0 (fertile, non-irradiated females and males), xxGy. $w$ Tei: $w$ Suz $\times$ × Tei ô (fertile $w$ Suz females crossed with irradiated $w$ Tei males)]. Pupae were irradiated at 45, 60 and $90 \mathrm{~Gy}$. All adults used for the crosses were 5-6 days old. A GLMM (binomial family) analysis was performed to determine the differences between the crosses. alpha $=0.05$
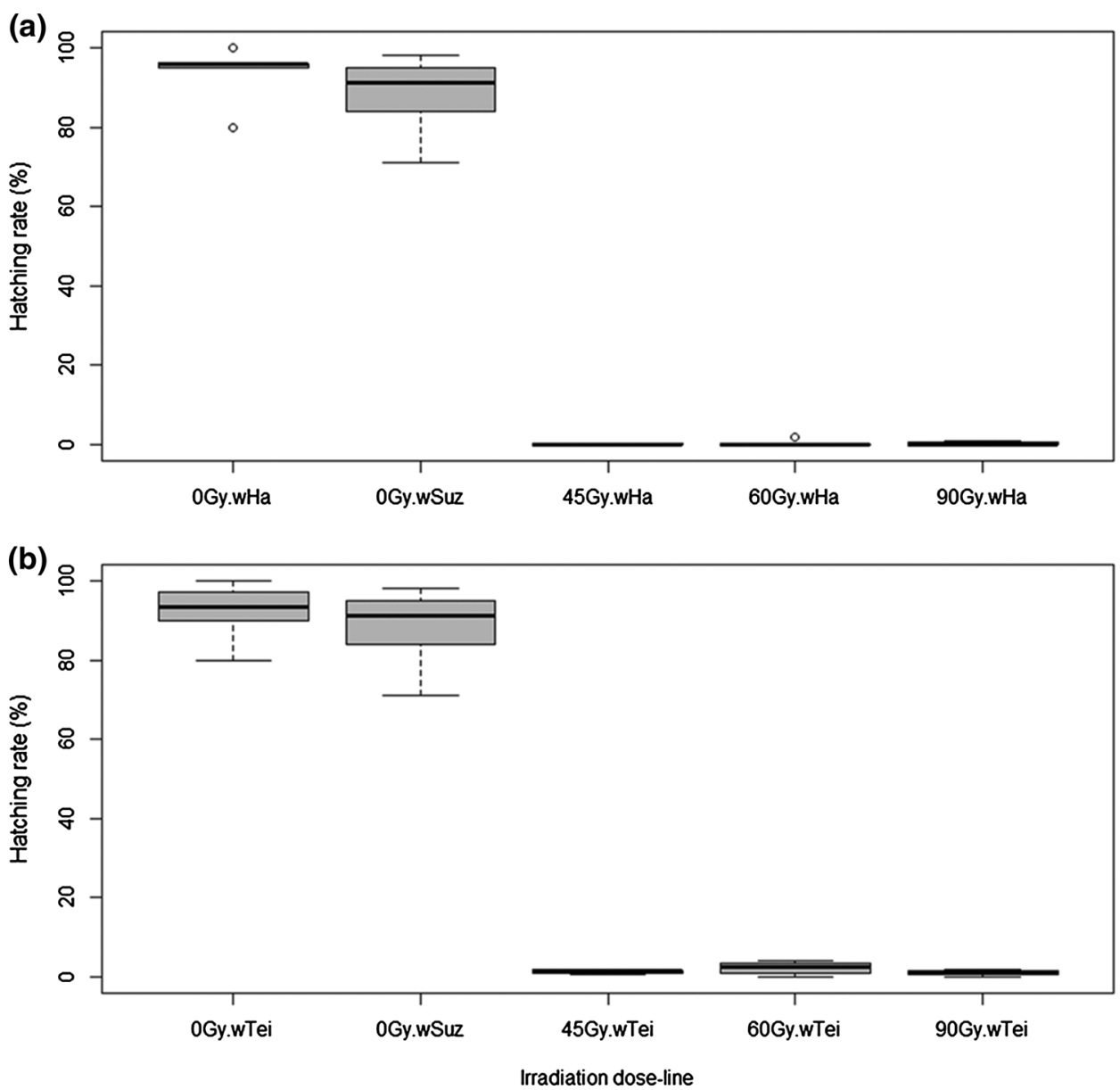

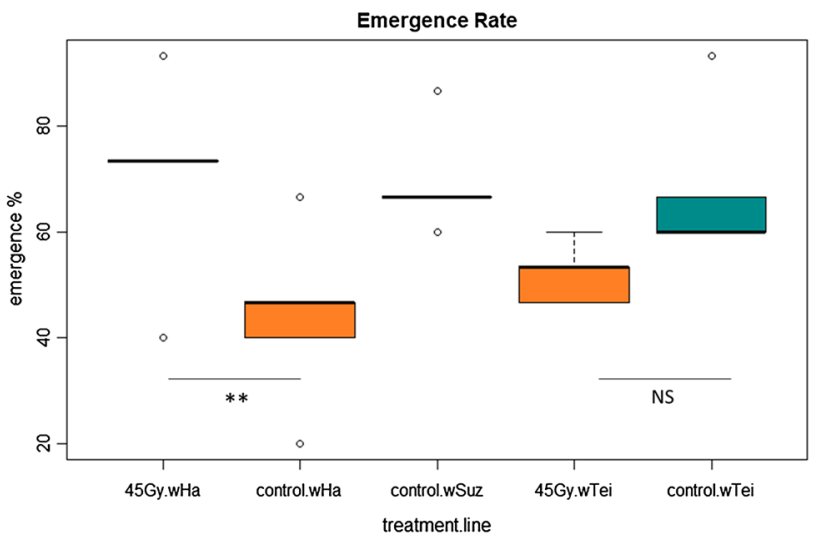

Fig. 5 Effect of 45 Gy irradiation dose on the emergence rate of irradiated and non-irradiated pupae. A GLMM (binomial family) analysis was performed to determine the differences between the groups. ${ }^{* *} p<.01$ (Kruskal-Wallis Chi-squared $=11.21, d f=4, p$ value $=0.0243, N=75$ )

on the Wolbachia strain rather than the host species. On the other hand, the host nuclear background is actively involved in the expression of fitness costs or benefits within the same species and it can be a leading factor in delivering decisive Wolbachia phenotypes (Dean 2006; Mouton et al. 2007; Poinsot et al. 1998; Veneti et al. 2012). In this study, we assessed the biological traits of two Wolbachia-infected $D$. suzukii lines which are maintained under a different genetic background than the one they were developed in. Our results clearly demonstrated that fecundity, hatch rate, sex ratio and adult longevity are not affected by the infection status. Mazzetto et al. (2015) have previously reported a beneficial effect of $w$ Suz infection on female fecundity compared to antibiotic-treated individuals which was not confirmed by our study. On the contrary, we observed a negative impact of the $w \mathrm{Ha}$ and $w$ Tei infections on the pupal weight. The pupal weight can be used as a proxy to estimate the adult size (Nash and Chapman 2014). Low pupal weight might indicate weak adults with decreased flight ability (FAO/ IAEA/USDA 2014) that could undermine the success of a management program. Through our single-pair crosses we determined the CI levels of $w \mathrm{Ha}$ and $w$ Tei lines and showed that $w \mathrm{Ha}$ induces strong $\mathrm{CI}$ in $D$. suzukii, but $w$ Tei CI levels are rather moderate. The $w$ Tei results are not in alignment with the study performed by Cattel et al. (2018), in which a high level of CI was induced by the $w$ Tei-infected $D$. suzukii 

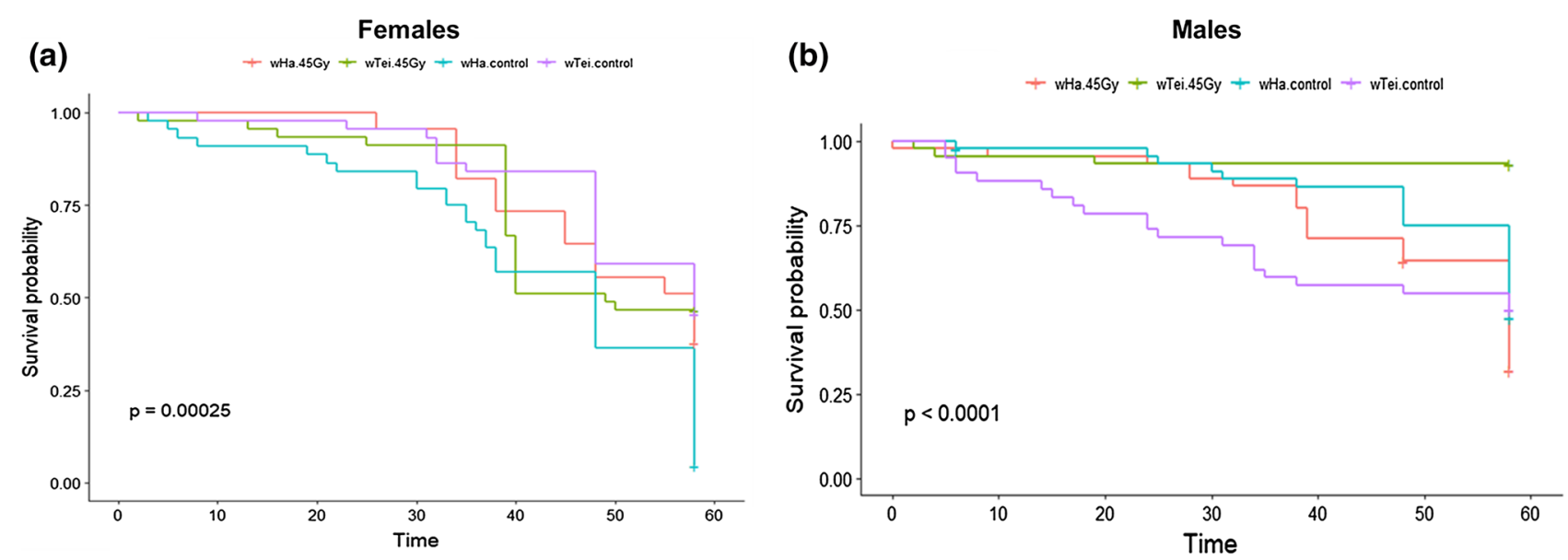

Fig. 6 Effect of 45 Gy irradiation dose on a female and $\mathbf{b}$ male longevity. Flies were provided with an agar-sugar substrate and dead flies were recorded daily. Significant differences were measured with a log-rank test. The $x$-axis represents time in days

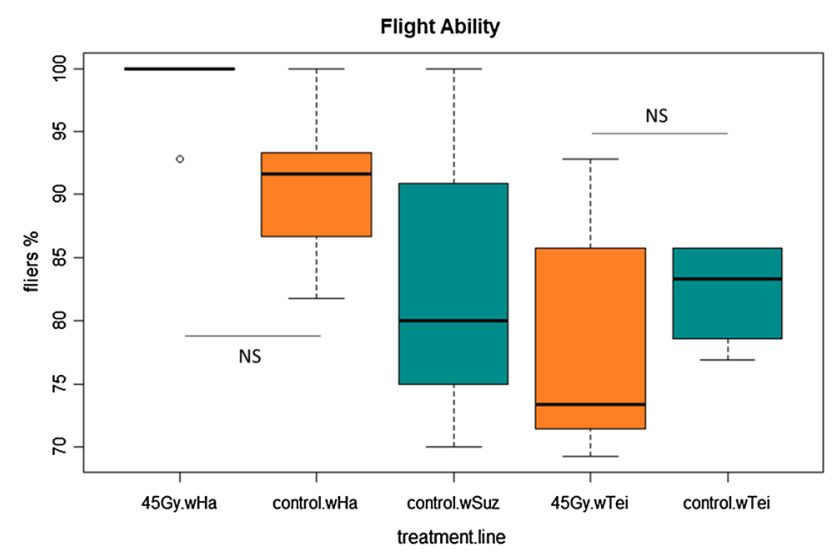

Fig. 7 Effect of 45 Gy irradiation dose on the flight ability of irradiated and non-irradiated adults. A GLMM (binomial family) analysis was performed to determine the differences between the groups

line $\left(96.46 \% \mathrm{CI}_{\text {corr }}\right.$ for the uninfected females). The observed differences could be due to the alternate host genetic background used in the two studies. These effects might be proved to be detrimental for the biological quality of the host (Calvitti et al. 2010; Fraser et al. 2017), and the cost-benefit evaluation will guide the decision for the use of a specific strain in large-scale applications. The profound influence of the $D$. suzukii genetic background on the CI expression indicates that the host-bacterial symbiotic association should be first and foremost characterized before a potential IIT application. As a result, the SIT presents an advantage over the IIT on the grounds that in the SIT the insects from the targeted field population can be mass-reared, sterilized and released in the area, thus surpassing the barrier of the host genetic background. However, it is worth noting that SIT studies on $D$. suzukii that tested high irradiation doses on flies with different genetic backgrounds provided comparable results (Krüger et al. 2018; Lanouette et al. 2017; Sassù et al. 2019). In the study of Krüger and colleagues, the flies were collected in Brazil while the other two studies used flies from the same colony reared at the IPCL (Italian genetic background). Despite the wide range of irradiation doses tested in all the above studies, the results did not show any major discrepancies both for males and females.

Young males can induce high CI levels but the increasing age exhibits an apparent and rapid decline in CI expression (Awrahman et al. 2014; Reynolds and Hoffmann 2002). This was also confirmed by our study and in the case of $w \mathrm{Ha}$, young males aged 2-3 days old seem to have a better performance in exhibiting a high CI profile, while the CI levels induced by $w$ Tei males aged 2-3 days and 5-6 days old do not vary. In our irradiation experiments, the $w \mathrm{Ha}$ and $w \mathrm{Tei}$ males were 5-6 days old compared to the 2-to-3-day-old males used in the CI experiments. The low egg hatching we observed in the combined irradiation/Wolbachia-infected cases (Table 1) could be explained by the male age that seems to negatively affect the $\mathrm{CI}_{\text {corr }}$ levels. Especially concerning the $w$ Tei males, none of the three irradiation doses tested were free of "escapers" that reached the adult stage, while in the case of the $w$ Ha males none of the hatched eggs reached the adult stage. The male age is a factor that should be considered in the logistics burden of any operational program with an IIT component for $D$. suzukii, since only young males that exhibit high CI levels should be released on a frequent basis.

Drosophila suzukii constitutes a continuously expanding threat and its exceptional biological traits have elevated the management of this pest into a challenge. The absence of a robust and adequate sexing system for $D$. suzukii renders IIT, as a stand-alone control method, an unattainable 
choice (Nikolouli et al. 2018). The currently available studies performed on SIT and D. suzukii by using irradiation in the range of 120-200 Gy are in favor of the feasibility of this approach. Further knowledge should be acquired regarding the male mating competitiveness, the longevity and the flight ability of the sterile males, particularly in the field, prior to its deployment (Vreysen et al. 2006; Zhang et al. 2016; Parker and Mehta 2007). As shown in the mosquito vector species Ae. albopictus, combining the SIT with IIT has the advantage of requiring significantly reduced levels of radiation which may not significantly affect the biological quality of the sterile males (Zhang et al. 2016; Zheng et al. 2019). The combined SIT/IIT also presents an advantage over the IIT since the radiation-induced sterility complements the one induced by Wolbachia infection. Based on these as well as on the data presented in this study where a range of 45-90 Gy irradiation doses was tested, the combined SIT/IIT may also worth consideration as an alternative approach for the population suppression of $D$. suzukii (Nikolouli et al. 2018). However, knowledge on male mating competitiveness will also be required prior to any small or large-scale application.

\section{Author contribution statement}

$\mathrm{KN}$ and $\mathrm{KB}$ designed the study. $\mathrm{KN}$ performed the experiments, did the data analysis and wrote the manuscript. All authors reviewed and provided constructive comments for this manuscript. All authors read and approved the final manuscript.

Acknowledgements This study was supported by the French National Research Agency (ANR-15-CE21-0017-01) and the Austrian Science Fund (FWF): I 2604-B25. The authors would like to thank Rui Pereira, Marc Vreysen and Carlos Cáceres for their support throughout this study. We also thank Julien Cattel for providing the protocol for the age of males' effect.

\section{Compliance with ethical standards}

Conflict of interest The authors declare that they have no conflict of interest.

Ethical approval This article does not contain any studies with animals performed by any of the authors.

Informed consent Informed consent was obtained from all co-authors included in the study.

Open Access This article is licensed under a Creative Commons Attribution 4.0 International License, which permits use, sharing, adaptation, distribution and reproduction in any medium or format, as long as you give appropriate credit to the original author(s) and the source, provide a link to the Creative Commons licence, and indicate if changes were made. The images or other third party material in this article are included in the article's Creative Commons licence, unless indicated otherwise in a credit line to the material. If material is not included in the article's Creative Commons licence and your intended use is not permitted by statutory regulation or exceeds the permitted use, you will need to obtain permission directly from the copyright holder. To view a copy of this licence, visit http://creativecommons.org/licenses/by/4.0/.

\section{References}

Apostolaki A, Livadaras I, Saridaki A, Chrysargyris A, Savakis C, Bourtzis K (2011) Transinfection of the olive fruit fly Bactrocera oleae with Wolbachia: towards a symbiont-based population control strategy. J Appl Entomol. https://doi.org/10.111 1/j.1439-0418.2011.01614.x

Asplen MK, Anfora G, Biondi A, Choi D (2015) Invasion biology of spotted wing Drosophila (Drosophila suzukii): a global perspective and future priorities. J Pest Sci. https://doi.org/10.1007/ s10340-015-0681-z

Atyame CM, Cattel J, Lebon C et al (2015) Wolbachia-based population control strategy targeting Culex quinquefasciatus mosquitoes proves efficient under semi-field conditions. PLoS ONE. https://doi.org/10.1371/journal.pone.0119288

Awrahman ZA, Champion de Crespigny F, Wedell N (2014) The impact of Wolbachia, male age and mating history on cytoplasmic incompatibility and sperm transfer in Drosophila simulans. J Evol Biol. https://doi.org/10.1111/jeb.12270

Bakri A, Mehta K, Lance DR (2005) Sterilizing insects with ionizing radiation. In: Dyck VA, Hendrichs J, Robinson AS (eds) Sterile insect technique: principles and practice in area-wide integrated pest management. Springer, Dordrecht, pp 233-269

Baldo L, Hotopp JCD, Jolley KA et al (2006) Multilocus sequence typing system for the endosymbiont Wolbachia pipientis. Appl Environ Microbiol. https://doi.org/10.1128/AEM.00731-06

Bates D, Mächler M, Bolker B, Walker S (2015) Fitting linear mixed-effects models using lme4. J Stat Softw. https://doi. org/10.18637/jss.v067.i01

Beers EH, Van SR, Shearer PW et al (2011) Developing Drosophila suzukii management programs for sweet cherry in the western United States. Pest Manag Sci. https://doi.org/10.1002/ps.2279

Bolda MP, Goodhue RE, Zalom FG (2010) Spotted wing Drosophila: potential economic impact of a newly established pest. ARE Update 13(3):5-8. University of California Giannini Foundation of Agricultural Economics. https://giannini.ucop.edu/publi cations/are-update/issues/2010/13/3/spotted-wing-drosophila-p/

Bourtzis K, Lees RS, Hendrichs J, Vreysen MJ (2016) More than one rabbit out of the hat: radiation, transgenic and symbiont-based approaches for sustainable management of mosquito and tsetse fly populations. Acta Trop. https://doi.org/10.1016/j.actatropic a.2016.01.009

Bruck DJ, Bolda M, Tanigoshi L et al (2011) Laboratory and field comparisons of insecticides to reduce infestation of Drosophila suzukii in berry crops. Pest Manag Sci. https://doi.org/10.1002/ ps. 2242

Calabria G, Máca J, Bächli G et al (2012) First records of the potential pest species Drosophila suzukii (Diptera: Drosophilidae) in Europe. J Appl Entomol. https://doi.org/10.111 1/j.1439-0418.2010.01583.x

Calkins CO, Parker AG (2005) Sterile insect quality. In: Dyck VA, Hendrichs J, Robinson AS (eds) Sterile insect technique: principles and practice in area-wide integrated pest management. Springer, Dordrecht, pp 269-296

Calvitti M, Moretti R, Lampazzi E, Bellini R, Dobson SL (2010) Characterization of a new Aedes albopictus (Diptera: Culicidae)-Wolbachia pipientis (Rickettsiales: Rickettsiaceae) 
symbiotic association generated by artificial transfer of the $w$ Pip strain from Culex pipiens (Diptera: Culicidae). J Med Entomol. https://doi.org/10.1603/me09140

Cattel J, Kaur R, Gibert P et al (2016) Wolbachia in European populations of the invasive pest Drosophila suzukii: regional variation in infection frequencies. PLoS ONE. https://doi. org/10.1371/journal.pone.0147766

Cattel J, Nikolouli K, Andrieux T et al (2018) Back and forth Wolbachia transfers reveal efficient strains to control spotted wing drosophila populations. J Appl Ecol. https://doi. org/10.1111/1365-2664.13101

Cayol JP, Hendrichs J, Enkerlin W, et al (2002) The sterile insect technique: an environment friendly method for the area-wide integrated management of insect pests of economic significance. In: Proceedings of the 2nd international conference on the alternative control methods against plant pests and diseases. Lille, France, pp 593-600

Cini A, Ioriatti C, Anfora G (2012) A review of the invasion of Drosophila suzukii in Europe and a draft research agenda for integrated pest management. Bull Insectol 65:149-160

Cuthbertson AG, Collins DA, Blackburn LF, Audsley N, Bell HA (2014) Preliminary screening of potential control products against Drosophila suzukii. Insects. https://doi.org/10.3390/ insects5020488

Cutler GC (2013) Insects, insecticides and hormesis: evidence and considerations for study. Dose Response. https://doi. org/10.2203/dose-response.12-008.Cutler

De Ros G, Conci S, Pantezzi T, Savini G (2015) The economic impact of invasive pest Drosophila suzukii on berry production in the Province of Trento, Italy. J Berry Res. https://doi. org/10.3233/JBR-150092

Dean MD (2006) A Wolbachia-associated fitness benefit depends on genetic background in Drosophila simulans. Proc Biol Sci. https ://doi.org/10.1098/rspb.2005.3453

Deprá M, Poppe JL, Schmitz HJ et al (2014) The first records of the invasive pest Drosophila suzukii in the South American continent. J Pest Sci. https://doi.org/10.1007/s10340-014-0591-5

Desneux N, Decourtye A, Delpuech JM (2007) The sublethal effects of pesticides on beneficial arthropods. Annu Rev Entomol. https ://doi.org/10.1146/annurev.ento.52.110405.091440

DiGiacomo G, Hadrich J, Hutchison WD et al (2019) Economic impact of spotted wing Drosophila (Diptera: Drosophilidae) yield loss on minnesota raspberry farms: a grower survey. J Integr Pest Manag. https://doi.org/10.1093/jipm/pmz006

Dos Santos LA, Mendes MF, Krüger AP et al (2017) Global potential distribution of Drosophila suzukii (Diptera Drosophilidae). PLoS ONE. https://doi.org/10.1371/journal.pone.0174318

Dyck VA, Hendrichs J, Robinson AS (2005) Sterile insect technique principles and practice in area-wide integrated pest management. Springer, Dordrecht, p 787p

FAO/IAEA/USDA (2014) Product quality control for sterile mass reared and released tephritid fruit flies, Version 6.0. http:// wwwnaweb.iaea.org/nafa/ipc/public/QualityControl.pdf. Accessed 18 July 2019

Fraimout A, Debat V, Fellous S et al (2017) Deciphering the routes of invasion of Drosophila suzukii by means of ABC random forest. Mol Biol Evol. https://doi.org/10.1093/molbev/msx050

Fraser JE, De Bruyne JT, Iturbe-Ormaetxe I et al (2017) Novel Wolbachia-transinfected Aedes aegypti mosquitoes possess diverse fitness and vector competence phenotypes. PLoS Pathog. https ://doi.org/10.1371/journal.ppat.1006751

Gafchromic ${ }^{\circledR}$ Dosimetry System for SIT (2004) Standard operating procedure v.1.1. Dosimetry system for SIT: manual for Gafchromic ${ }^{\circledR}$ film. http://www-naweb.iaea.org/nafa/ipc/publi c/ipc-gafchromic-dosimetry-sterile-insect-technique.html. Accessed 18 July 2019
Goodhue RE, Bolda M, Farnsworth D et al (2011) Spotted wing Drosophila infestation of California strawberries and raspberries: economic analysis of potential revenue losses and control costs. Pest Manag Sci. https://doi.org/10.1002/ps.2259

Grassi A, Giongo L, Palmieri L (2011) Drosophila (Sophophora) suzukii (Matsumura), new pest of soft fruits in Trentino (NorthItaly) and in Europe. IOBC/WPRS Bull 70:121-128

Guerfali MM, Parker A, Fadhl S et al (2012) Fitness and reproductive potential of irradiated mass-reared Mediterranean fruit fly males Ceratitis capitata (Diptera: Tephritidae): lowering radiation doses. Fla Entomol. https://doi.org/10.1653/024.094.0443

Hall TA (1999) BioEdit: a user-friendly biological sequence alignment editor and analysis program for Windows 95/98/NT. Nucl Acids Symp Ser 41:95-98

Hamby KA, Bellamy DE, Chiu JC et al (2016) Biotic and abiotic factors impacting development, behavior, phenology, and reproductive biology of Drosophila suzukii. J Pest Sci. https://doi. org/10.1007/s10340-016-0756-5

Hamm CA, Begun DJ, Vo A et al (2014) Wolbachia do not live by reproductive manipulation alone: infection polymorphism in Drosophila suzukii and D. Subpulchrella. Mol Ecol. https://doi. org/10.1111/mec.12901

Hauser M (2011) A historic account of the invasion of Drosophila suzukii (Matsumura) (Diptera: Drosophilidae) in the continental United States, with remarks on their identification. Pest Manag Sci. https://doi.org/10.1002/ps.2265

Haye T, Girod P, Cuthbertson AGS et al (2016) Current SWD IPM tactics and their practical implementation in fruit crops across different regions around the world. J Pest Sci 89:643-651. https ://doi.org/10.1007/s10340-016-0737-8

Hothorn T, Bretz F, Westfall P (2008) Simultaneous inference in general parametric models. Biom J. https://doi.org/10.1002/ bimj. 200810425

Hughes GL, Rasgon JL (2014) Transinfection: a method to investigate Wolbachia-host interactions and control arthropod-borne disease. Insect Mol Biol. https://doi.org/10.1111/imb.12066

Iglesias L, Liburd OE (2017) Identification of biorational insecticides for managing spotted wing drosophila in organic blueberry production. Acta Hortic. https://doi.org/10.17660/ActaHortic .2017 .1180 .38

Kaplan EL, Meier P (1958) Nonparametric estimation from incomplete observations. J Am Stat Assoc 53:457-481. https://doi. org/10.1080/01621459.1958.10501452

Knipling EF (1979) The basic principles of insect population suppression and management. Agriculture Handbook. USDA No. 512

Krüger AP, Schlesener DCH, Martins LN, Wollmann J, Deprá M, Garcia FRM (2018) Effects of irradiation dose on sterility induction and quality parameters of Drosophila suzukii (Diptera: Drosophilidae). J Econ Entomol. https://doi.org/10.1093/jee/tox349

Lanouette G, Brodeur J, Fournier F et al (2017) The sterile insect technique for the management of the spotted wing drosophila Drosophila suzukii: establishing the optimum irradiation dose. PLoS ONE. https://doi.org/10.1371/journal.pone.0180821

Laven H (1967) A possible model for speciation by cytoplasmic isolation in the Culex pipiens complex. Bull World Health Organ. https ://doi.org/10.1101/SQB.1959.024.01.017

Le Bourg É (2010) Combined effects of suppressing live yeast and of a cold pretreatment on longevity, aging and resistance to several stresses in Drosophila melanogaster. Biogerontology. https://doi. org/10.1007/s10522-009-9250-2

Lee JC, Bruck DJ, Curry H et al (2011) The susceptibility of small fruits and cherries to the spotted-wing drosophila, Drosophila suzukii. Pest Manag Sci. https://doi.org/10.1002/ps.2225

Lees RS, Gilles JR, Hendrichs J et al (2015) Back to the future: the sterile insect technique against mosquito disease vectors. Curr Opin Insect Sci. https://doi.org/10.1016/j.cois.2015.05.011 
Lenth RV (2016) Least-squares means: the R package_-lsmeans. J Stat Softw. https://doi.org/10.18637/jss.v069.i01

Mains JW, Kelly PH, Dobson KL et al (2019) Localized control of Aedes aegypti (Diptera: Culicidae) in Miami, FL, via inundative releases of Wolbachia-infected male mosquitoes. J Med Entomol. https://doi.org/10.1093/jme/tjz051

Martinez J, Tolosana I, Ok S et al (2017) Symbiont strain is the main determinant of variation in Wolbachia-mediated protection against viruses across Drosophila species. Mol Ecol. https://doi. org/10.1111/mec.14164

Mazzetto F, Gonella E, Alma A (2015) Wolbachia infection affects female fecundity in Drosophila suzukii. Bull Insectol 68(1):153-157

Mazzi D, Bravin E, Meraner M et al (2017) Economic impact of the introduction and establishment of Drosophila suzukii on sweet cherry production in Switzerland. Insects. https://doi.org/10.3390/ insects 8010018

Monnin D, Kremer N, Berny C, Henri H, Dumet A, Voituron Y, Desouhant E, Vavre F (2016) Influence of oxidative homeostasis on bacterial density and cost of infection in Drosophila-Wolbachia symbioses. J Evol Biol. https://doi.org/10.1111/jeb.12863

Mouton L, Henri H, Charif D, Boulétreau M, Vavre F (2007) Interaction between host genotype and environmental conditions affects bacterial density in Wolbachia symbiosis. Biol Lett. https://doi. org/10.1098/rsbl.2006.0590

Munhenga G, Brooke BD, Gilles JRL et al (2016) Mating competitiveness of sterile genetic sexing strain males (GAMA) under laboratory and semi-field conditions: steps towards the use of the Sterile Insect Technique to control the major malaria vector Anopheles arabiensis in South Africa. Parasit Vectors. https://doi. org/10.1186/s13071-016-1385-9

Nash WJ, Chapman T (2014) Effect of dietary components on larval life history characteristics in the medfly (Ceratitis capitata: Diptera Tephritidae). PLoS ONE. https://doi.org/10.1371/journ al.pone.0086029

Nikolouli K, Colinet H, Renault D et al (2018) Sterile insect technique and Wolbachia symbiosis as potential tools for the control of the invasive species Drosophila suzukii. J Pest Sci. https://doi. org/10.1007/s10340-017-0944-y

O'Connor L, Plichart C, Sang AC et al (2012) Open release of male mosquitoes infected with a Wolbachia biopesticide: field performance and infection containment. PLoS Negl Trop Dis. https:// doi.org/10.1371/journal.pntd.0001797

Parker A, Mehta K (2007) Sterile insect technique: a model for dose optimisation for improved sterile insect quality. Florida Entomol. https://doi.org/10.1653/0015-4040(2007)90\%5b88:sitam $\mathrm{f} \% 5 \mathrm{~d} 2.0 . \mathrm{co}$

Pereira R, Yuval B, Liedo P et al (2013) Improving sterile male performance in support of programmes integrating the sterile insect technique against fruit flies. J Appl Entomol. https://doi.org/10.1 111/j.1439-0418.2011.01664.x

Poinsot D, Bourtzis K, Markakis G et al (1998) Wolbachia transfer from Drosophila melanogaster into D. simulans: host effect and cytoplasmic incompatibility relationships. Genetics 150(1):227-237

Poyet M, Le Roux V, Gibert P et al (2015) The wide potential trophic niche of the asiatic fruit fly Drosophila suzukii: the key of its invasion success in temperate Europe? PLoS ONE. https://doi. org/10.1371/journal.pone.0142785

R Core Team (2018) R: a language and environment for statistical computing. R Foundation for Statistical Computing, Vienna. https ://www.R-project.org/. Accessed 16 June 2019
Reynolds KT, Hoffmann AA (2002) Male age, host effects and the weak expression or non-expression of cytoplasmic incompatibility in Drosophila strains infected by maternally transmitted Wolbachia. Genet Res. https://doi.org/10.1017/s0016672302005827

Rota-Stabelli O, Blaxter M, Anfora G (2013) Drosophila suzukii. Curr Biol. https://doi.org/10.1016/j.cub.2012.11.021

Roubos CR, Rodriguez-Saona C, Holdcraft R et al (2014) Relative toxicity and residual activity of insecticides used in blueberry pest management: mortality of natural enemies. J Econ Entomol. https ://doi.org/10.1603/EC13191

Rull J, Biotecnologia P, Díaz-Fleischer F, Arredondo J (2007) Irradiation of Anastrepha ludens (Diptera: Tephritidae) revisited: optimizing sterility induction. J Econ Entomol. https://doi. org/10.1603/0022-0493(2007)100\%5b1153:ioaldt\%5d2.0.co;2

Sánchez-Ramos I, Fernández CE, González-Núñez M (2019a) Comparative analysis of thermal performance models describing the effect of temperature on the preimaginal development of Drosophila suzukii. J Pest Sci. https://doi.org/10.1007/s10340-018-1030-9

Sánchez-Ramos I, Gómez-Casado E, Fernández CE, González-Núñez M (2019b) Reproductive potential and population increase of Drosophila suzukii at constant temperatures. Entomol Gen. https ://doi.org/10.1127/entomologia/2019/0794

Santoiemma G, Trivellato F, Caloi V et al (2019) Habitat preference of Drosophila suzukii across heterogeneous landscapes. J Pest Sci. https://doi.org/10.1007/s10340-018-1052-3

Sassù F, Nikolouli K, Pereira R, Vreysen MJB, Stauffer C, Cáceres C (2019) Irradiation dose response under hypoxia for the application of the sterile insect technique in Drosophila suzukii. PLOS ONE. https://doi.org/10.1371/journal.pone.0226582

Sial AA, Roubos CR, Gautam BK et al (2019) Evaluation of organic insecticides for management of spotted-wing drosophila (Drosophila suzukii) in berry crops. J Appl Entomol. https://doi. org/10.1111/jen.12629

Tait G, Grassi A, Pfab F, Crava CM (2018) Large-scale spatial dynamics of Drosophila suzukii in Trentino, Italy. J Pest Sci. https://doi. org/10.1007/s10340-018-0985-x

Therneau T (2015) A package for survival analysis in S. R package. http://CRAN.R-project.org/package $=$ survival. Accessed 16 June 2019

Tochen S, Dalton DT, Wiman N et al (2014) Temperature-related development and population parameters for Drosophila suzukii (Diptera: Drosophilidae) on cherry and blueberry. Environ Entomol. https://doi.org/10.1603/EN13200

Tochen S, Woltz JM, Dalton DT et al (2016) Humidity affects populations of Drosophila suzukii (Diptera: Drosophilidae) in blueberry. J Appl Entomol. https://doi.org/10.1111/jen.12247

Toledo J, Rull J, Oropeza A et al (2004) Irradiation of Anastrepha obliqua (Diptera: Tephritidae) revisited: optimizing sterility induction. J Econ Entomol. https://doi.org/10.1093/jee/97.2.383

Van Timmeren S, Isaacs R (2013) Control of spotted wing drosophila, Drosophila suzukii, by specific insecticides and by conventional and organic crop protection programs. Crop Prot. https://doi. org/10.1016/j.cropro.2013.08.003

Veneti Z, Zabalou S, Papafotiou G et al (2012) Loss of reproductive parasitism following transfer of male-killing Wolbachia to Drosophila melanogaster and Drosophila simulans. Heredity (Edinb). https://doi.org/10.1038/hdy.2012.43

Vreysen MJB, Hendrichs J, Enkerlin WR (2006) The sterile insect technique as a component of sustainable area-wide integrated pest management of selected horticultural insect pests. Res Inst Pomol Floric J Fruit Ornam Plant Res 14:107-132 
Walsh DB, Bolda MP, Goodhue RE et al (2011) Drosophila suzukii (Diptera: Drosophilidae): invasive pest of ripening soft fruit expanding its geographic range and damage potential. J Integr Pest Manag. https://doi.org/10.1603/IPM10010

Werren JH, Windsor DM (2000) Wolbachia infection frequencies in insects: evidence of a global equilibrium? Proc Biol Sci. https:// doi.org/10.1098/rspb.2000.1139

Wong JS, Cave AC, Lightle DM et al (2018) Drosophila suzukii flight performance reduced by starvation but not affected by humidity. J Pest Sci. https://doi.org/10.1007/s10340-018-1013-x

Zabalou S, Riegler M, Theodorakopoulou M et al (2004) Wolbachiainduced cytoplasmic incompatibility as a means for insect pest population control. Proc Natl Acad Sci U S A. https://doi. org/10.1073/pnas.0403853101

Zhang D, Zheng X, Xi Z, Bourtzis K, Gilles JR (2015a) Combining the sterile insect technique with the incompatible insect technique: I-impact of Wolbachia infection on the fitness of triple- and double-infected strains of Aedes albopictus. PLoS ONE. https://doi. org/10.1371/journal.pone.0121126
Zhang D, Lees RS, Xi Z et al (2015b) Combining the sterile insect technique with Wolbachia-based approaches: II—a safer approach to Aedes albopictus population suppression programmes, designed to minimize the consequences of inadvertent female release. PLoS ONE. https://doi.org/10.1371/journal.pone.0135194

Zhang D, Lees RS, Xi Z et al (2016) Combining the sterile insect technique with the incompatible insect technique: III-robust mating competitiveness of irradiated triple Wolbachia-infected Aedes albopictus males under semi-field conditions. PLoS ONE. https ://doi.org/10.1371/journal.pone.0151864

Zheng X, Zhang D, Li Y, Yang C et al (2019) Incompatible and sterile insect techniques combined eliminate mosquitoes. Nature. https ://doi.org/10.1038/s41586-019-1407-9

Publisher's Note Springer Nature remains neutral with regard to jurisdictional claims in published maps and institutional affiliations. 